(C)1982. The Genetical Society of Great Britain

\title{
EFFECT OF YEASTS ON POLYMORPHISM AT THE Adh LOCUS IN DROSOPHILA MELANOGASTER
}

\author{
S. P. DOWNES and J. M. THODAY \\ Department of Genetics, Downing Street, Cambridge CB2 3EH, U.K.
}

Received 31.iii.82

\section{SUMMARY}

\begin{abstract}
Thirty-six populations were founded from equal numbers of females and males from ten pairs of isofemale lines of Drosophila melanogaster and maintained on standard medium. They were divided into four sets of 9 , each set being provided with a different yeast. Six enzyme polymorphisms and the $\operatorname{In}(3 R) P$ inversion polymorphism were scored. No effect of yeast type was evident except for the alcohol dehydrogenase locus. This showed evidence for strong balancing selection in populations maintained with Metschnikowia pulcherrima, but not with Saccharomyces cerevisiae.
\end{abstract}

\section{INTRODUCTION}

YEAST is one of the more important components of the environments of Drosophilids. Indeed Starmer (1981) provides evidence that the spectrum of yeast species is a major component determining the distribution of Drosophila species. Further, natural populations may grow on a variety of yeasts which differ in their capacity to support the flies. For instance, Ali and El-Helw (1974) demonstrated that larvae of Egyptian populations of $D$. melanogaster and $D$. simulans preferentially chose different yeast species from a mixture of species in their natural habitats. They also showed that the larvae of wild type and a vg laboratory strain of $D$. melanogaster differed in yeast preference, and that the relative productivity of females of the two strains also differed with yeast. Preference and productivity were not correlated. A striking feature of their results was that of the yeasts used, species of Candida and Nadsonia supported their wild type Drosophila better than the standard laboratory yeast, Saccharomyces cerevisiae even though the larvae never chose Nadsonia when offered a choice.

It therefore seemed worthwhile testing whether these particular yeasts differentially affect the maintenance of protein polymorphisms, for most laboratory experiments on polymorphism have been made using $S$. cerevisiae to supplement the medium and other yeasts might give different results.

\section{MATERIALS AND METHODS}

(i) Origin of flies

The populations were founded from the OK lines. These lines were established from 10 females collected together by J. N. Thompson in Oklahoma, the first progeny of each female being split to set up a pair of isofemale lines. The allele and inversion frequencies in these lines are given in table 1. 


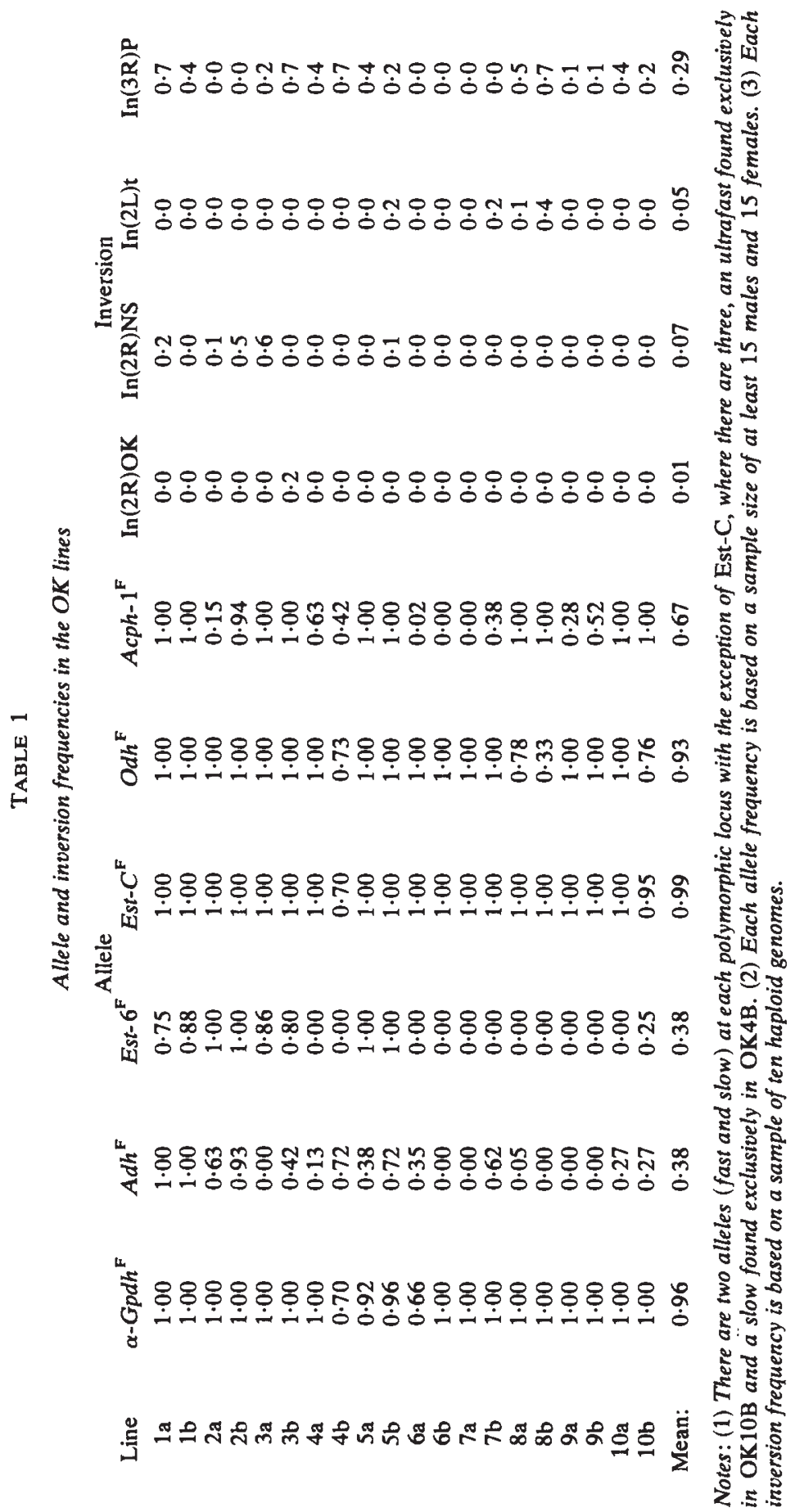




\section{(ii) Establishment and maintenance of populations}

The experimental lines were established as follows: 10 bottle populations (OKY1-10) were set up with ten of each sex from each of the OK lines. At the next generation each bottle was used to found four bottle populations one on each of the following yeasts, obtained from the National Collection of Yeast Cultures: (Stocks 166 and 471 are synonymous with two of the "Candida" species of Ali and El-Helw).

$\begin{array}{cl}\text { NCYC Stock No. } & \\ 166 & \text { Metschnikowia pulcherrima } \\ 378 & \text { Nadsonia elongata var. anomala } \\ 471 & \text { Hansenula fulvescens } \\ 1335 & \text { Saccharomyces cerevisiae }\end{array}$

The experiment started in early 1974 , and the populations were maintained by mass transfer thereafter. The populations were maintained at $25^{\circ} \mathrm{C}$ with transfer every two weeks, except that for a period of one year they were maintained at $18^{\circ} \mathrm{C}$ with transfer every three weeks. When the populations were returned to $25^{\circ} \mathrm{C}$ a replicate set was kept at $18^{\circ} \mathrm{C}$. (Tests of these replicates gave similar gene frequencies to those from the main lines so that this $18^{\circ} \mathrm{C}$ period does not seem to have influenced the results.) Oatmeal-molasses-agar medium was used for the first two years. Since then Harris instant medium has been used. Of course, other microorganisms occur in the culture medium as well as the desired species of yeast. Nevertheless, inoculation with the required fresh yeast every two weeks must have ensured that the desired yeast was predominant, or at least frequent, in the culture media. One population, OKY10-166, was lost early in the experiment. Analyses have therefore been restricted to 36 populations, nine supplemented with each type of yeast.

\section{(iii) Assays}

After approximately 120 generations (April 1979) ten adults of each sex from each population were each assayed for six loci $(\alpha-G p d h, A d h$, Est-6, Est-C, Odh and Acph-1). The sampling was repeated after approximately 145 generations (March 1980).

Forty chromosomes from every population were assayed for $\operatorname{In}(3 R) P$ between late March and early July 1980. Presence or absence of the inversion was ascertained from the progeny of individual $F_{1}$ females, one only from any OKY parent, backcrossed to a homozygous red $e$ stock (description of mutants in Lindsley and Grell, 1968). In(3R)P almost completely eliminates recombinants between these loci.

\section{RESULTS}

Allele and inversion frequencies in the OK lines provide one estimate of the initial frequencies in the OKY populations, though it should be borne in mind that the OK lines were sampled over four years after the OKY populations were founded, so that the estimates may not be accurate if some loci in the OK lines changed frequency during that period. However, a comparison of inbreeding coefficients (see Cavalli-Sforza, 1966) gives no 


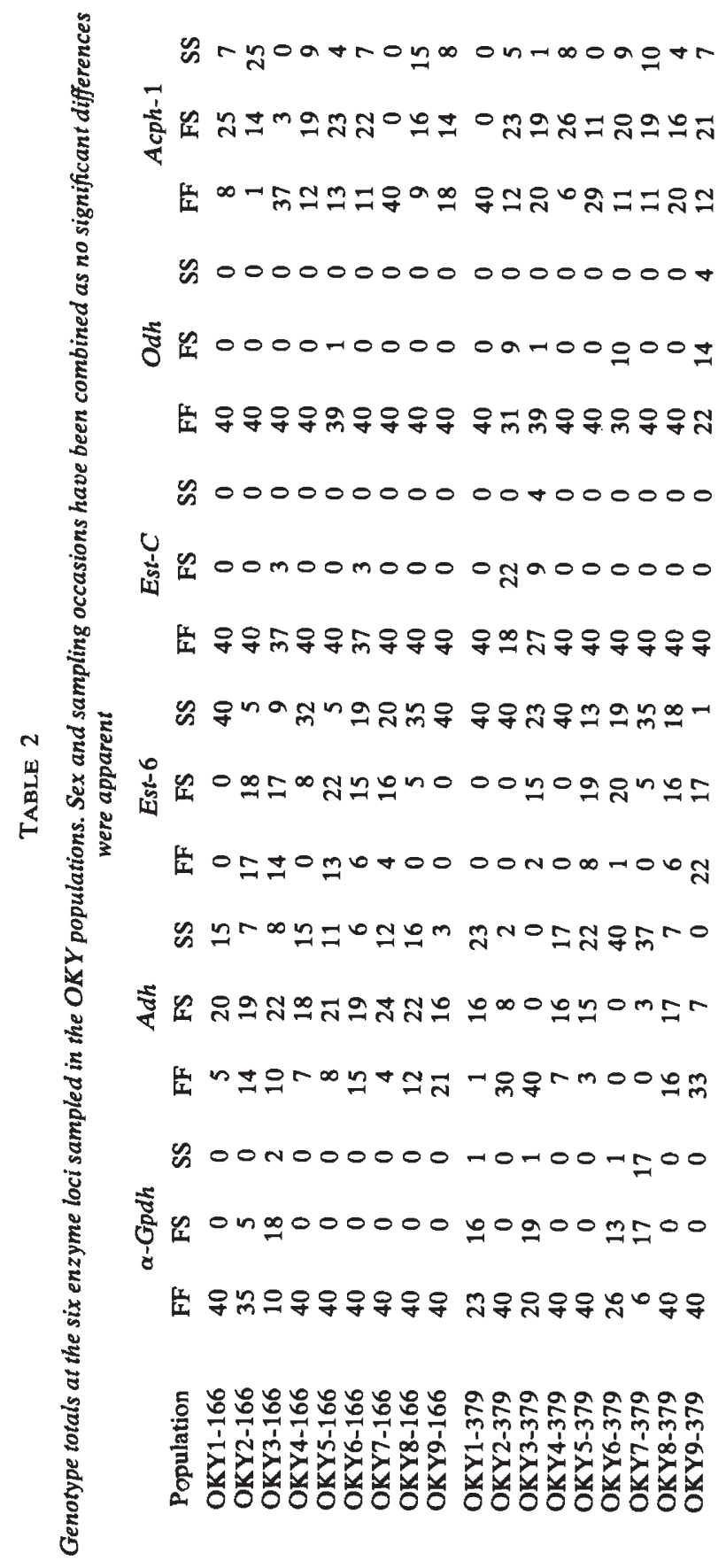




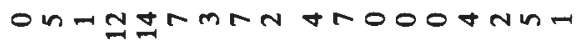

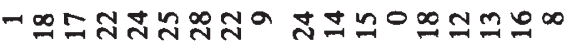

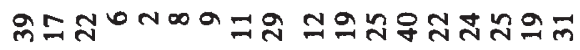
$0000000+00000-10000$ OOONOON=O OOMONROOOO

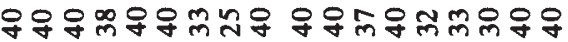
$00 N 000000000000000$ 000000000000000000

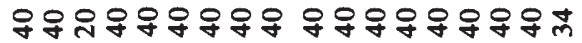

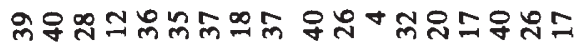
-ON

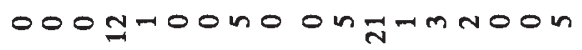

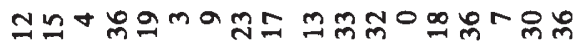

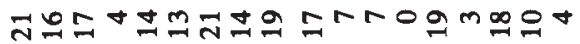
ragor OONOOOHOO OnHOHHHJm

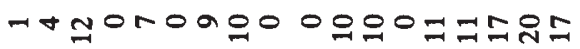

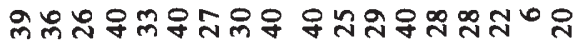

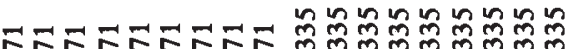
守守年守守 خัخ

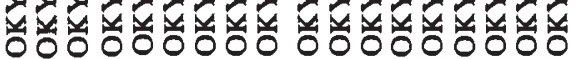


indication that selection affected the loci differentially in these OK isofemale lines. The genotype frequencies for the experimental lines are listed in table 2 and the gene frequencies, mean gene frequencies, $\sigma_{p}^{2}$ and $\mathrm{F}$ computed from these are given in table 3.

TABLE 3

Allele frequencies at the six enzyme loci and $\operatorname{In}(3 R) P$ frequency in the OKY populations. Sexes and sampling occasions have been combined

\begin{tabular}{|c|c|c|c|c|c|c|c|}
\hline Population & $\alpha-G p d h^{F}$ & $A d h^{F}$ & $E s t-6^{\mathrm{F}}$ & $E s t-C^{\mathbf{F}}$ & $O d h^{\mathrm{F}}$ & $A c p h-1^{F}$ & $\operatorname{In}(3 R) P$ \\
\hline OKY1-166 & 1.0000 & $0 \cdot 3750$ & 0.0000 & 1.0000 & 1.0000 & 0.5125 & $0 \cdot 150$ \\
\hline OKY2-166 & 0.9375 & 0.5875 & 0.6500 & 1.0000 & $1 \cdot 0000$ & $0 \cdot 2000$ & $0 \cdot 150$ \\
\hline OKY3-166 & 0.4750 & 0.5250 & 0.5625 & 0.9625 & 1.0000 & 0.9625 & 0.175 \\
\hline OKY4-166 & $1 \cdot 0000$ & 0.4000 & 0.1000 & 1.0000 & 1.0000 & 0.5375 & 0.675 \\
\hline OKY5-166 & 1.0000 & 0.4625 & 0.6000 & 1.0000 & 0.9875 & 0.6125 & 0.650 \\
\hline OKY6-166 & $1 \cdot 0000$ & 0.6125 & 0.3375 & 0.9625 & 1.0000 & 0.5500 & 0.700 \\
\hline OKY7-166 & 1.0000 & 0.4000 & $0 \cdot 3000$ & 1.0000 & 1.0000 & 1.0000 & 0.650 \\
\hline OKY8-166 & $1 \cdot 0000$ & 0.5750 & 0.0625 & 1.0000 & 1.0000 & 0.4250 & 0.225 \\
\hline OKY9-166 & $1 \cdot 0000$ & 0.7250 & 0.0000 & $1 \cdot 0000$ & 1.0000 & 0.6250 & 0.475 \\
\hline & 0.9347 & 0.5181 & $0 \cdot 2903$ & 0.9917 & 0.9986 & 0.6028 & 0.4278 \\
\hline$\sigma$ & 0.0268 & 0.0124 & 0.0620 & 0.0002 & 0.0000 & 0.0552 & 0.0551 \\
\hline $\mathrm{F}$ & 0.4391 & 0.0497 & 0.3009 & 0.0243 & - & 0.2305 & 0.2251 \\
\hline OKY1-379 & 0.7750 & $0 \cdot 2250$ & $0 \cdot 0000$ & 1.0000 & 1.0000 & 1.0000 & 1.000 \\
\hline-379 & $1 \cdot 0000$ & 0.8500 & 0.0000 & 0.7250 & 0.8875 & & \\
\hline OKY3-379 & 0.7375 & 1.0000 & 0.2375 & 0.7875 & 0.9875 & 0.7375 & 0.700 \\
\hline OKY4-379 & $1 \cdot 0000$ & $0 \cdot 3750$ & 0.0000 & 1.0000 & 1.0000 & 0.4750 & 0.575 \\
\hline OKY5-379 & 1.0000 & 0.2625 & 0.4375 & 1.0000 & 0.7875 & 0.8625 & 0.225 \\
\hline OKY6-379 & 0.8125 & 0.0000 & $0 \cdot 2750$ & 1.0000 & 0.8750 & 0.5250 & $0 \cdot 125$ \\
\hline OKY7-379 & 0.3625 & 0.0375 & 0.0625 & 1.0000 & 1.0000 & 0.5125 & .400 \\
\hline OKY8-379 & $1 \cdot 0000$ & 0.6125 & $0 \cdot 3500$ & 1.0000 & 1.0000 & 0.7000 & 0.500 \\
\hline OKY9-379 & $1 \cdot 0000$ & 0.9125 & 0.7625 & 1.0000 & 0.7000 & 0.5625 & 0.500 \\
\hline & 0.8542 & 0.4750 & $0 \cdot 2361$ & 0.9458 & 0.9153 & 0.6625 & 0.5028 \\
\hline$\sigma^{2}$ & 0.0411 & 0.1290 & 0.0588 & 0.0105 & 0.0110 & 0.0281 & 0.0580 \\
\hline $\mathrm{F}$ & $0 \cdot 3300$ & 0.5173 & $0 \cdot 3260$ & 0.2048 & 0.1419 & 0.1257 & 0.2320 \\
\hline OKY1-471 & 0.9875 & 0.4375 & 0.0125 & 1.0000 & 1.0000 & 0.9875 & 1.000 \\
\hline OKY & 00 & 0.4250 & 00 & 1.0000 & 1.0000 & & \\
\hline OKY3-471 & $0 \cdot 8000$ & 0.6875 & $0 \cdot 1500$ & 0.7250 & 1.0000 & 0.7625 & 0.500 \\
\hline OKY4-471 & 1.0000 & 0.0500 & 0.5000 & 1.0000 & 0.9750 & 0.4250 & $0 \cdot 275$ \\
\hline OKY5-471 & 0.9125 & $0 \cdot 3500$ & 0.0625 & 1.0000 & 1.0000 & 00 & 0.550 \\
\hline OKY6-471 & 1.0000 & 0.7625 & 0.0625 & 1.0000 & 1.0000 & 0.5125 & 0.675 \\
\hline OK & 0.7875 & 0.5125 & 0.0375 & 1.0000 & 0.9125 & & 0.500 \\
\hline OKY8-471 & 0.8750 & $0 \cdot 2500$ & 0.3 & 1.0000 & 0.7625 & 0.5 & 0.075 \\
\hline OKY9-471 & 1.0000 & 0.3375 & 0.0375 & 1.0000 & 1.0000 & 0.8375 & 0.550 \\
\hline & 0.9236 & 0.4236 & 0.1333 & 0.9694 & 0.9611 & 0.6275 & 0.4917 \\
\hline$\sigma_{p}^{2}$ & 0.0065 & 0.0417 & 0.0264 & 0.0075 & 0.0057 & 0.0369 & 0.0618 \\
\hline . & 0.0921 & $0 \cdot 1708$ & 0.2285 & 0.2528 & 0.1525 & 0.1579 & 0.2473 \\
\hline OKY1-1355 & $1 \cdot 0000$ & 0.4625 & 0.0000 & 1.0000 & 1.0000 & 0.6000 & 0.600 \\
\hline & & & & & 1.0000 & & \\
\hline OKY3-1355 & $0 \cdot 8500$ & $0 \cdot 1125$ & 0.7125 & 1.0000 & 0.9625 & 0.8125 & 0.700 \\
\hline OKY4-1355 & 1.0000 & 1.0000 & 0.1125 & 1.0000 & 1.0000 & & 0.675 \\
\hline OK & 0.8375 & 0.3125 & 0.28 & 1.0000 & 0.8875 & 0.7750 & 0.350 \\
\hline OKY6-1355 & 0.8375 & 0.0625 & 0.31 & 1.0000 & 0.9125 & & 0.275 \\
\hline OK & 0.7625 & 0.6000 & 0.0000 & 1.0000 & 0.8750 & 0.7875 & 0.225 \\
\hline OKY8-1355 & 0.4000 & 0.1250 & 0.1750 & 1.0000 & 1.0000 & 0.6 & 0.275 \\
\hline OKY9-1355 & 0.7125 & 0.0500 & $0 \cdot 3500$ & & 1.0000 & & 0.400 \\
\hline $\bar{P}$ & 0.7944 & $0 \cdot 3125$ & 0.2431 & 0.9917 & 0.9597 & 0.7694 & 0.4222 \\
\hline & 0.0285 & 0.0928 & 0.0421 & 0.0006 & 0.0025 & 0.0132 & 0.0306 \\
\hline$F$ & $0 \cdot 1745$ & 0.4319 & 0.2288 & 0.0729 & 0.0646 & 0.0744 & 0.1254 \\
\hline
\end{tabular}



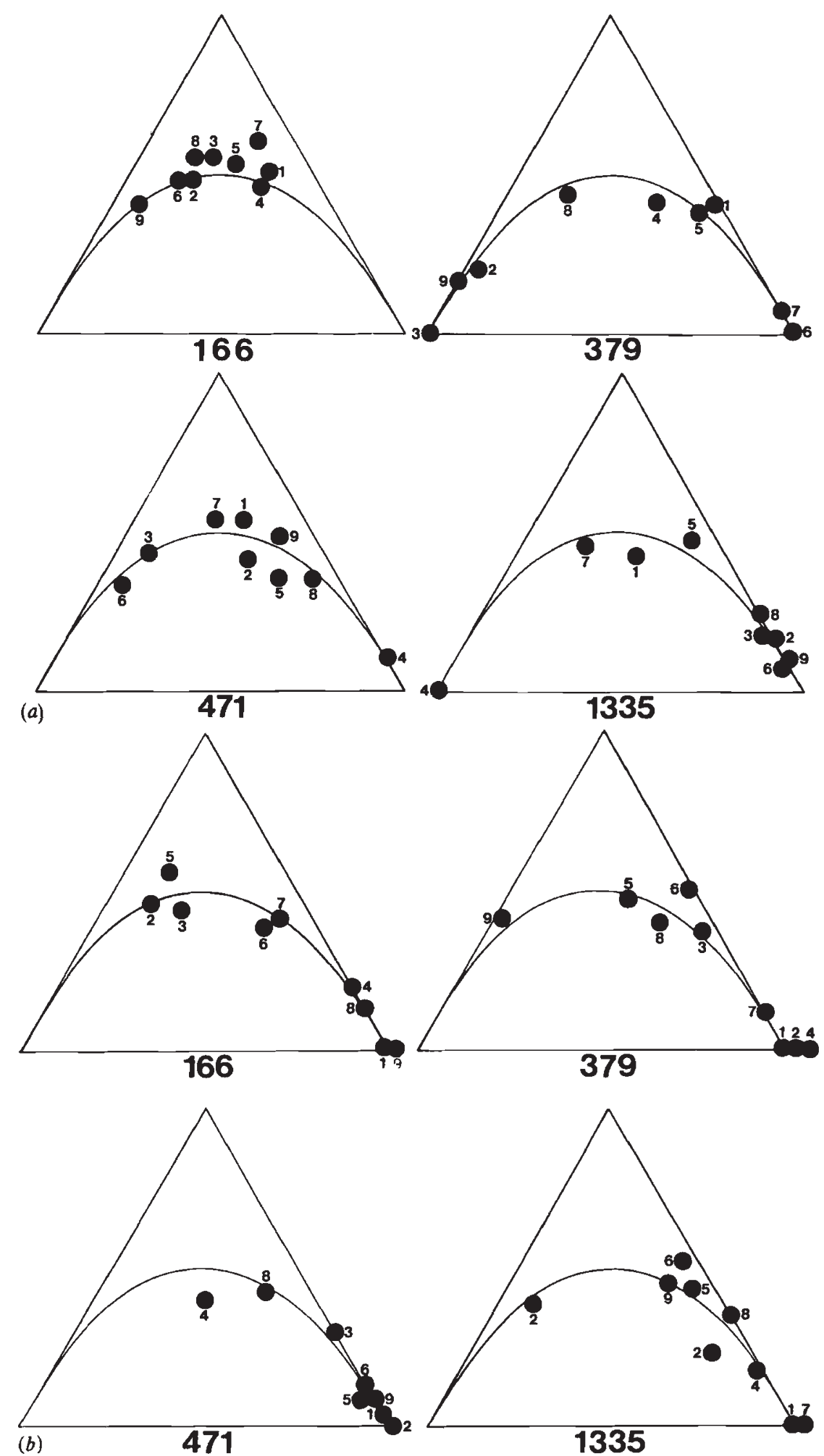

FIG. 1.-Genotype frequencies at the (a) Adh, (b) Est-6 and (c) Acph-1 loci in OKY populations. 


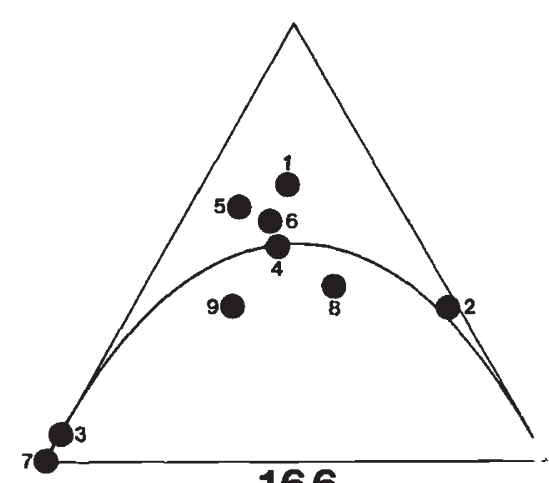

166

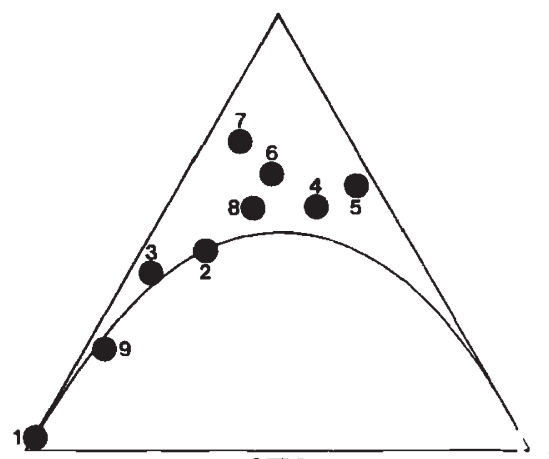

(c)
471
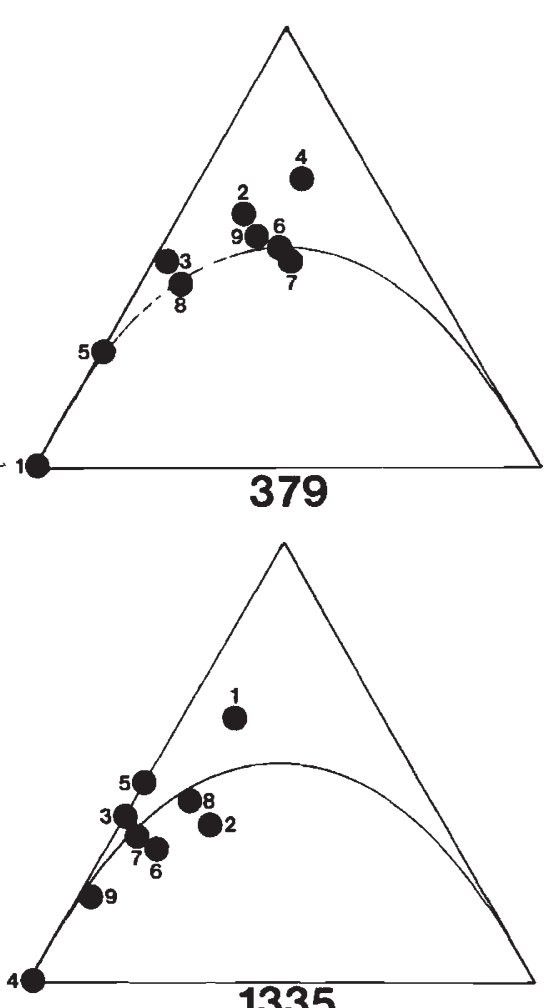

1335

FIG. 1.-continued

The Adh, Est-6 and Acph-1 loci each started with two alleles at appreciable frequencies and so provide more information than the other loci. The data from table 2 for these loci have accordingly been plotted on de Finetti diagrams (Cannings and Edwards, 1968). The pattern of variation at the $A d h$ locus among populations maintained with 166 yeast is strikingly different from the $A d h$ pattern on other yeasts, and from the Est- 6 and $A c p h-1$ patterns (fig. 1). These 166 populations cluster around the $(0 \cdot 25$, $0.50,0.25$ ) point for $A d h$, reflecting gene frequencies close to 0.5 and a low variance of genotype and allele frequencies.

To determine whether the variances of genotype frequencies differ, a $\chi^{2}$ may be calculated for the nine populations maintained on medium supplemented with any yeast, thus measuring the heterogeneity of genotype frequencies at any locus. These $\chi^{2}$ values, calculated by combining the two least frequent genotypic classes if necessary, and the associated mean squares are presented in table 4. The mean squares for $\boldsymbol{A d h}$ genotypes among populations maintained on medium supplemented with the four yeasts are compared in table 5 . The variance among populations maintained on 166 is significantly lower than that of populations maintained on medium supplemented with the other yeasts. The variance for populations maintained on 471 is significantly lower than that of those maintained on medium supplemented with 379 or 1335 , confirming the impression given by fig. 1 that the pattern of variation among the 471 populations is intermediate 
TABLE 4

$\chi^{2}$ values measuring the heterogeneity of genotype numbers for all possible combinations of locus and yeast

\begin{tabular}{|c|c|c|c|c|}
\hline Locus & Yeast & $\mathrm{df}$ & $x^{2}$ & M.S. \\
\hline$\alpha-G p d h$ & $\begin{array}{r}166 \\
379 \\
471 \\
1335\end{array}$ & $\begin{array}{l}- \\
8 \\
8 \\
8\end{array}$ & $\begin{array}{r}- \\
171.6295 \\
56.1769 \\
95.9884\end{array}$ & $\begin{array}{r}-\overline{4537} \\
7 \cdot 0221 \\
11.9986\end{array}$ \\
\hline$A d h$ & $\begin{array}{r}166 \\
379 \\
471 \\
1335\end{array}$ & $\begin{array}{l}16 \\
16 \\
16 \\
16\end{array}$ & $\begin{array}{r}40 \cdot 0228 \\
299 \cdot 2229 \\
120 \cdot 0871 \\
285 \cdot 5238\end{array}$ & $\begin{array}{r}2 \cdot 5014 \\
18 \cdot 7014 \\
7 \cdot 5054 \\
17 \cdot 8452\end{array}$ \\
\hline Est-6 & $\begin{array}{r}166 \\
379 \\
471 \\
1335\end{array}$ & $\begin{array}{r}16 \\
8 \\
8 \\
8\end{array}$ & $\begin{array}{l}184 \cdot 5966 \\
170 \cdot 8857 \\
117 \cdot 2504 \\
115 \cdot 6992\end{array}$ & $\begin{array}{l}11 \cdot 5373 \\
21 \cdot 3607 \\
14 \cdot 6563 \\
14 \cdot 4624\end{array}$ \\
\hline Acph-1 & $\begin{array}{r}166 \\
379 \\
471 \\
1335\end{array}$ & $\begin{array}{r}16 \\
16 \\
16 \\
8\end{array}$ & $\begin{array}{r}182 \cdot 7843 \\
102 \cdot 9435 \\
138 \cdot 5260 \\
53 \cdot 0879\end{array}$ & $\begin{array}{r}11.4240 \\
6.4340 \\
8.6579 \\
6.6360\end{array}$ \\
\hline
\end{tabular}

\section{TABLE 5}

Variance ratios comparing the heterogeneity of genotype numbers at the Adh locus among populations maintained on the four types of yeast. Degrees of freedom are given in table 4

\begin{tabular}{|c|c|c|c|c|}
\hline & & & er mean sq & \\
\hline & & 471 & 1335 & 379 \\
\hline $\begin{array}{l}\text { smaller } \\
\text { mean }\end{array}$ & $\begin{array}{r}166 \\
471 \\
1325\end{array}$ & $\begin{array}{c}3 \cdot 0005^{*} \\
-\end{array}$ & $\begin{array}{l}7 \cdot 1340^{* * *} \\
2 \cdot 3776^{*}\end{array}$ & $\begin{array}{l}7.4763^{* * *} \\
2.4917^{*} \\
1.0480^{\mathrm{n} . \mathrm{s}}\end{array}$ \\
\hline $\begin{array}{r}* 0.05 \\
* * 0.01 \\
* * * P<0 \\
\text { n.s. not si }\end{array}$ & 01. & & & \\
\hline
\end{tabular}

between that for the 166 populations and those for the 379 and 1335 populations. The mean squares for loci among populations maintained on medium supplemented with 166 yeast have been compared in table 6 . The variance of genotype numbers at the Adh locus is significantly lower than that of those of the Est-6 or Acph-1 loci. Moreover, the variance of genotype numbers at the $A d h$ locus among populations maintained on

\section{TABLE 6}

Variance ratios comparing the heterogeneity of genotype numbers at three loci among populations maintained on medium supplemented with 166 yeast. Degrees of freedom are given in table 4

\begin{tabular}{lccc} 
& & \multicolumn{2}{c}{ larger mean square } \\
& & Acph-1 & Est-6 \\
Smaller & Adh & $4.5670^{* *}$ & $4.6123^{* *}$ \\
mean & & & \\
square $\quad$ Acph -1 & & $1.0099^{\text {n.s. }}$ \\
** $0.01>P>0.001$. & \\
n.s. not significant.
\end{tabular}


medium supplemented with 166 yeast is significantly lower than any other combination of yeast and locus. The $A d h$ locus has clearly been less affected by inbreeding in the 166 treated populations than it has been in populations maintained on medium supplemented with other yeasts, and also than have other loci in the 166 treated populations. This can only be the result of some kind of selection, and since the mean $A d k^{F}$ frequencies are similar among populations maintained on medium supplemented with each of the yeasts, it must be some form of balancing selection. The variance provides no evidence whether such selection affected other loci or affected $A d h$ in populations on the yeasts 379 or 1335 ; the alleles could be effectively neutral in these conditions or, if they were affected by balancing selection, it must have been much weaker than that affecting $A d h$ on 166 (and 471).

In addition to this balancing selection there is a suggestion that Est-6 has been affected by directional selection, for the Est $6^{\mathrm{F}}$ frequency is lower in populations maintained on all four yeasts than the initial frequency estimated from the OK lines. There are no striking differences at the $\alpha-G p d h, E s t-C$ and $O d h$ loci, but with allele frequencies initially so close to fixation the evidence is weak.

The estimates of $\operatorname{In}(3 \mathrm{R}) \mathrm{P}$ frequency among populations maintained on medium supplemented with each of the four yeasts are very similar, and higher than the initial frequency estimate from the OK lines, which estimate is based on examination of only ten chromosomes in each OK line. However, the calculated variances of inversion frequency in the OKY lines do not suggest that inbreeding has affected the inversion less than it has affected allozyme polymorphisms, other than the $A d h$ polymorphism on 166. The balancing selection for $A d h$ on yeast 166 must have been stronger than any there was affecting inversion 3R Payne. We had expected that this inversion, which is so common in natural populations, might have given lower inbreeding coefficients than the allozyme polymorphisms. That it has not, could be regarded as evidence that Est-6 and $A c p h-1$ as well as $A d h$ on 379 and 1335 are also affected by balancing selection.

\section{Possible nature of balance selection for $\boldsymbol{A D H}$}

The low between-population variance of $A d h$ frequency on 166 could result either from heterozygous advantage or frequency-dependent selection or a combination of the two. Although five populations are above the Hardy-Weinberg parabola in the Finetti diagram (fig. 1) indicating an excess of heterozygotes, none of the eight possible $\chi^{2}$ tests of goodness of fit with Hardy-Weinberg genotype frequencies are significant. An increased sample of 150 individuals was assayed from OKY5-166, but no excess of heterozygotes was detected. If heterozygous advantage were the mechanism responsible for, or contributing to, the maintenance of the polymorphism, selection must be too weak to detect in this way, at least at the gene frequencies in the lines.

The experimental design of Kojima and Tobari (1969) was used to test for frequency-dependent selection. Two populations descended from a common founder, OKY5-166 and OKY5-1335 were chosen, and ten homozygous lines, five of each of $A d h^{\mathrm{FF}}$ and $A d h^{\text {ss }}$, were extracted from each population by pair-mating males and virgin females. To extract $A d h^{\mathrm{FF}}$ lines from OKY5-1335, two rounds of pair-matings had to be made. 
These lines were used to produce mixed progenies at two levels of egg input: the high (H) with $60 A d h^{\mathrm{FF}}: 30 A d h^{\mathrm{FS}}: 10 A d h^{\mathrm{SS}}$ females, and the low (L) with $10 A d h^{\mathrm{FF}}: 30 A d h^{\mathrm{FS}}: 60 A d h^{\text {SS }}$ females per culture. The heterozygous eggs were laid by equal numbers of females from the two reciprocal crosses. 16 experimental cultures were set up, each population being tested at each input level on each yeast, the whole being replicated twice. While the parents were being collected they were kept on medium supplemented with the yeast that was to be used in the experimental culture, so a population to be tested on a novel yeast could become to some extent free of its routine yeast. The eggs were laid in standard culture bottles supplemented with either 166 or 1335 yeast, the females being allowed to lay for two days. Twelve days later a sample of 200 adults was taken. The results are presented in table 7 .

TABLE 7

Results of the frequency-dependent selection experiment

(i) $H$ input

\begin{tabular}{|c|c|c|c|c|c|c|}
\hline Population & Yeast & $\begin{array}{c}\text { Replicate } \\
\text { number }\end{array}$ & Mortality & $A d h^{\mathbf{F F}}$ & $\begin{array}{l}\text { output } \\
A_{d} h^{\mathrm{FS}}\end{array}$ & $A d h^{\text {ss }}$ \\
\hline 166 & 166 & 1 & 2 & 114 & 62 & 24 \\
\hline 166 & $\begin{array}{r}166 \\
1335\end{array}$ & 2 & 0 & 90 & 84 & 26 \\
\hline 166 & $\begin{array}{l}1335 \\
1335\end{array}$ & $\begin{array}{l}1 \\
2\end{array}$ & $\begin{array}{l}1 \\
2\end{array}$ & 114 & $\begin{array}{l}62 \\
64\end{array}$ & $\begin{array}{l}24 \\
24\end{array}$ \\
\hline $\begin{array}{r}166 \\
1335\end{array}$ & & $\begin{array}{l}2 \\
1\end{array}$ & $\begin{array}{l}2 \\
8\end{array}$ & 112 & $\begin{array}{l}64 \\
68\end{array}$ & $\begin{array}{l}24 \\
42\end{array}$ \\
\hline $\begin{array}{l}1335 \\
1335\end{array}$ & $\begin{array}{l}166 \\
166\end{array}$ & $\begin{array}{l}1 \\
2\end{array}$ & $\begin{array}{l}8 \\
5\end{array}$ & $\begin{array}{r}90 \\
103\end{array}$ & $\begin{array}{l}68 \\
63\end{array}$ & $\begin{array}{l}42 \\
34\end{array}$ \\
\hline 1335 & 1335 & 1 & 7 & 126 & 50 & 24 \\
\hline 1335 & 1335 & 2 & 5 & 122 & 59 & 19 \\
\hline Totals & & & & $\overline{871}$ & $\overline{512}$ & 217 \\
\hline \multicolumn{7}{|c|}{ (ii) $L$ input } \\
\hline 166 & 166 & 1 & 0 & 19 & 72 & 109 \\
\hline 166 & 166 & 2 & 5 & 14 & 74 & 112 \\
\hline 166 & 1335 & 1 & 0 & 23 & 100 & 77 \\
\hline 166 & 1335 & 2 & 2 & 34 & 71 & 95 \\
\hline 1335 & 166 & 1 & 6 & 28 & 91 & 81 \\
\hline 1335 & 166 & 2 & 3 & 24 & 79 & 97 \\
\hline 1335 & 1335 & 1 & 4 & 23 & 65 & 112 \\
\hline 1335 & 1335 & 2 & 0 & 25 & 72 & 103 \\
\hline Totals & & & & $\overline{190}$ & $\overline{624}$ & 786 \\
\hline
\end{tabular}

The ratio of output of adults of each genotype to its expected input in each population on each yeast, combining replicates, is shown in fig. 2 . The results of a variance test of $\chi^{2}$ values, for each input level, are given in table 8. The major source of heterogeneity at the high input is associated with the different yeasts. The output from cultures supplemented with 1335 yeast is close to the expected input, but for the cultures supplemented with 166 yeast there is a clear excess of the rarer classes.

When $A d h^{\mathrm{F}}$ is at the low input level, the between-yeasts source of variation is not significant but there is a significant flies $\times$ yeast item, indicating that the populations have responded differently to the two yeasts. The 166 population shows evidence of frequency-dependent selection when tested on 1335 yeast but not on 166 yeast, whereas the reverse is true for the 1335 population (fig. 2). There appears to be an excess of heterozygotes 


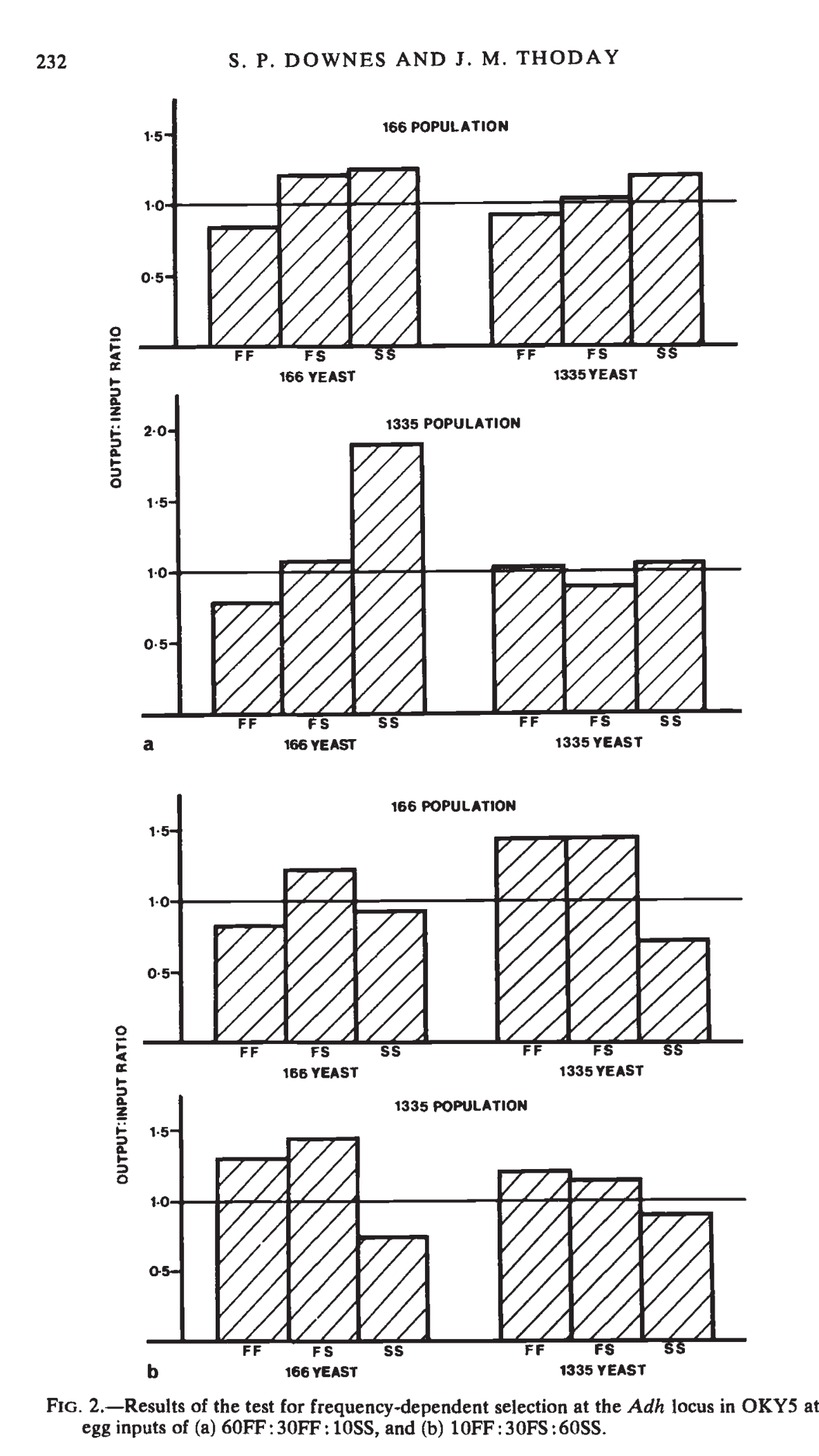$$
\text { 等, }
$$ 
TABLE 8

Analysis of $\chi^{2}$ for the Adh frequency-dependent experiment results

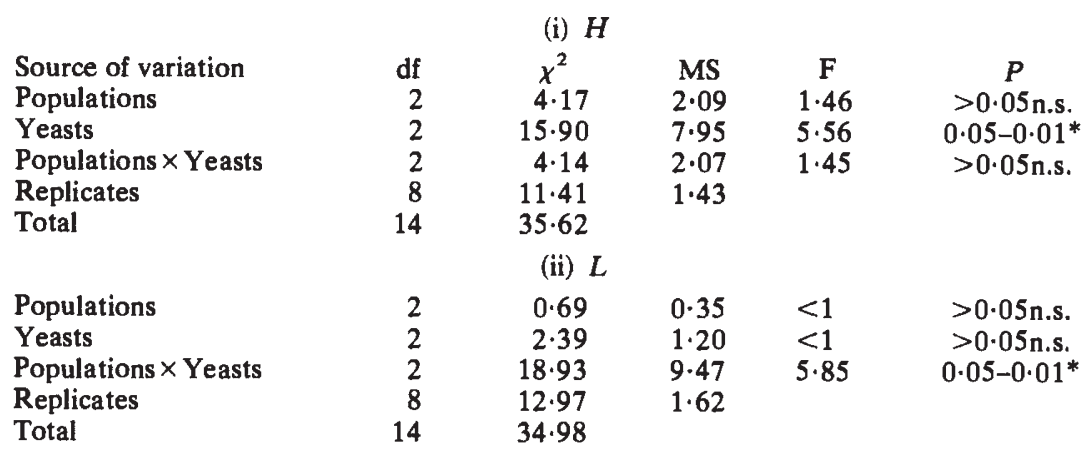

at this input level. The results suggest that frequency-dependent selection may be important at high $A d h^{\mathrm{F}}$ frequencies, whereas at low $A d h^{\mathrm{F}}$ frequencies heterozygous advantage is more important.

\section{Discussion}

Evidence for the selective effects of yeast on protein polymorphisms has been rather indirect. Powell (1971) and McDonald and Ayala (1974) found that cage populations provided with a variety of environmental factors, including yeasts, maintained a higher degree of heterozygosity than cage populations in more uniform environments. However, the design and analysis of these experiments did not resolve the specific selective mechanisms responsible for the observed correlations. In a more thoroughly designed population cage experiment, Oakeshott (1979) found that various yeasts differed in their selective effects on two loci, Amy and Idh-NADP in D. melanogaster.

The present results show that the maintenance of the Adh polymorphism is affected by yeast. The results do not provide evidence that selection is not contributing to the maintenance of the $A d h$ polymorphism on other yeasts, nor to that of the other enzyme and inversion polymorphisms. But selection must be invoked to explain the results obtained for the $A d h$ locus in populations maintained on medium supplemented with the yeast Metschnikowia pulcherrima.

We cannot of course be sure that the selection we have demonstrated acts on the Adh locus itself, but three lines of evidence suggest that the Adh locus may well be the target for selection: (a) ten genomes from OKY5-166 were free of inversions so there is no evidence that selection via $\operatorname{In}(2 \mathrm{~L}) \mathrm{t}$ can explain the results; (b) the experiment had run for 120 generations before the first assays so any initial disequilibria should have decayed to a considerable degree; (c) there appear to be selective effects at the $A d h$ locus detectable in one generation in a population previously exposed to 1335 yeast when tested on 166 yeast (table 8).

We are aware that the tests for frequency-dependent selection we have done are open to some criticism (see Prout, 1971) and that the fact that the two input frequencies gave quite different results indicates that it would 
be unwise to conclude much about relative roles of heterosis and frequencydependent selection in maintaining both $A d h$ alleles on yeast 166.

But our results clearly indicate that conclusions based on experiments using Saccharomyces cerevisiae only must be of limited generality. And it should be noted the yeasts used in the experiment are of species which are common in the ecology of an Egyptian natural population (Ali and El-Helw, 1974), and that Melschnikowia pulcherrima (166) was one of those yeasts that gave higher productivity than Saccharomyces cerevisiae in their experiments.

Acknowledgements.-We are indebted to $\mathrm{Mr} \mathrm{R}$. Ison for maintaining the yeast cultures, to Miss P. Angel for maintaining the OK and OKY lines, and the S.R.C. for a grant which has aided this work. S.P.D. acknowledges an S.R.C. Studentship, and J.M.T. S.R.C. grant GR/A/3758.1.

\section{REFERENCES}

ALI, A. M. M., AND EL-HELW, M. R. 1974. Differences in the yeasts preferred by Drosophila melanogaster and D. simulans. Egypt. J. Genet. Cytol., 3, 204-210.

CANNINGS, C., AND EDWARDS, A. W. F. 1968. Natural selection and the de Finetti diagam. Ann. Hum. Genet., 31, 421-428.

CAVAlli-SFORZA, L. L. 1966. Population structure and human evolution. Proc. Roy. Soc. B., 164, 362-379.

KOJIMA, K., AND TOBARI, Y. N. 1969. The pattern of viability changes associated with genotype frequency at the alcohol dehydrogenase locus in Drosophila melanogaster. Genetics, 61, 201-209.

LINDSLEY, D. L., AND GRELL, E. H. 1968. Genetic variations of Drosophila melanogaster. Carnegie Institution of Washington publication no. 627.

MCDONALD, J. F., AND A YALA, F. J. 1974. Genetic response to environmental heterogeneity. Nature, 250, 572-574.

OAKESHOTT, J. G. 1979. Selection affecting enzyme polymorphisms in laboratory populations of Drosophila melanogaster. Oecologia (Berl.), 143, 341-354.

POWELL, J. R. 1971. Genetic polymorphism in varied environments. Science, 174, 1035-1036.

PROUT, T. 1971. The relation between fitness components and population prediction in Drosophila. I. The estimation of fitness components. Genetics, 68, 127-149.

STARMER, W. T. 1981. A comparison of Drosophila habitats according to the physiological attributes of the associated yeast communities. Evolution, 35, 38-52. 